Supporting Information for:

\title{
Shape and Composition Effects on Photocatalytic Hydrogen Production for Pt-Pd Alloy Cocatalysts
}

\author{
Muhua Luo ${ }^{1 \#}$, Pan $\mathrm{Lu}^{1 \#}$, Weifeng Yao ${ }^{1 *}$, Cunping Huang ${ }^{2}$, Qunjie $\mathrm{Xu}^{1^{*}}$, Qiang \\ $\mathrm{Wu}^{1}$, Yasutaka Kuwahara ${ }^{3}$ and Hiromi Yamashita ${ }^{3}$ \\ ${ }^{1}$ Shanghai Key Laboratory of Materials Protection and Advanced Materials in Electric \\ Power, College of Environmental \& Chemical Engineering, Shanghai University of \\ Electric Power, Shanghai 200090, P. R. China \\ ${ }^{2}$ Aviation Fuels Research Lab, FAA William J. Hughes Technical Center, Atlantic City \\ International Airport, NJ 08405, USA \\ ${ }^{3}$ Division of Materials and Manufacturing Science, Graduate School of Engineering, \\ Osaka University, 2-1 Yamada-oka, Suita, Osaka 565-0871, Japan \\ \#These authors contributed equally to this study and share first authorship \\ ${ }^{*}$ Corresponding author: yaoweifeng@shiep.edu.cn and xuqunjie@,shiep.edu.cn
}




\section{Materials and equipment:}

All chemicals and solutions in this research were used as received without further purification. These included: sodium tetrachloropalladate $\left(\mathrm{Na}_{2} \mathrm{PdCl}_{4}\right.$, Aladdin, $\left.\geq 99.9 \%\right)$, potassium tetrachloroplatinate $\left(\mathrm{K}_{2} \mathrm{PtCl}_{4}\right.$, Aladdin, $\geq 99.9 \%$ ), potassium iodide (KI, Aladdin, $\geq 99.5 \%$ ), sodium chloride ( $\mathrm{NaCl}$, Aladdin, $\geq 99.5 \%$ ), polyvinylpyrrolidone (PVP, K30, molar mass $=30,000)$ and $\mathrm{N}, \mathrm{N}$-dimethylformamide (DMF, Aladdin, $\geq 99.9 \%$ ), cadmium sulfide (CdS, Energy Chemical, $\geq$ $98 \%$ ), ammonium sulfite monohydrate $\left(\left(\mathrm{NH}_{4}\right)_{2} \mathrm{SO}_{3}\right.$, Aladdin, $\left.\geq 92 \%\right)$, perchloric acid $\left(\mathrm{HClO}_{4}\right.$, Aladdin, $\geq 72 \%$ ) and $5 \%$ Nafion solution (Alpha). Water (18.3 M $\Omega-\mathrm{cm})$ for all experiments was prepared via an ultra-pure water purification system.

TEM and high resolution TEM (HRTEM) images and the selected area energy dispersive spectra (EDS) were obtained using a JEM-2100 transmission electron microscope equipped with a Link's EDS detector operated at $200 \mathrm{KV}$. X-ray diffraction (XRD) patterns were collected on a BRUKER-D8 X-ray diffractometer using $\mathrm{Cu} \mathrm{K} \alpha$ radiation $(0.15418 \mathrm{~nm})$. UV-Visible diffusion reflectance spectra were recorded using a UV-Visble spectrometer (UV-2550, Shimadzu) and were converted to absorption spectra by the standard Kubelka-Munk method. XPS analyses were performed on an ESCALAB250 spectrometer equipped with a monochromatized Al Ka (1486.6 $\mathrm{eV}$ ) source. $\mathrm{Pd} \mathrm{K}$-edge and $\mathrm{Pt}_{2}$-edge X-ray absorption fine structure (XAFS) data were collected in fluorescence mode at room temperature at BL01B1 facility with an attached $\mathrm{Si}(111)$ monochromator at SPring-8 (JASRI), Hyogo, Japan. For the Fourier transforms of extended X-ray absorption fine structure (EXAFS), the XAFS data were normalized by fitting the background absorption coefficient and the oscillations were extracted in the range of $4.0<k\left(\AA^{-1}\right)<12.0$ for Pd $\mathrm{K}$-edge and $4.0<k\left(\AA^{-1}\right)<10.0$ for $\mathrm{Pt}_{3}$-edge to obtain the radial structure function (RDF). All the EXAFS analysis was performed with Rigaku REX2000 software. 


\section{Loading Pt-Pd nanoparticles onto CdS photocatalyst}

$0.25 \mathrm{mg}$ as-prepared Pt-Pd nanocubes (NCs) or nanooctahedra (NOTa) were added into $100 \mathrm{~mL}$ deionized water containing $0.05 \mathrm{~g}$ commercial CdS photocatalyst. The resulting suspension solution was stirred for $2 \mathrm{~h}$ at room temperature. After centrifugation, the Pt-Pd/CdS loaded composites were washed with high purity water and dried at $60{ }^{\circ} \mathrm{C}$ under a vacuum. UV-Vis absorption spectra (Fig. S1) indicate that there is no characterized absorption of Pt-Pd nanoparticles in the filtrate, indicating that the Pt-Pd nanoparticles have been completely deposited onto the surface of CdS particles.

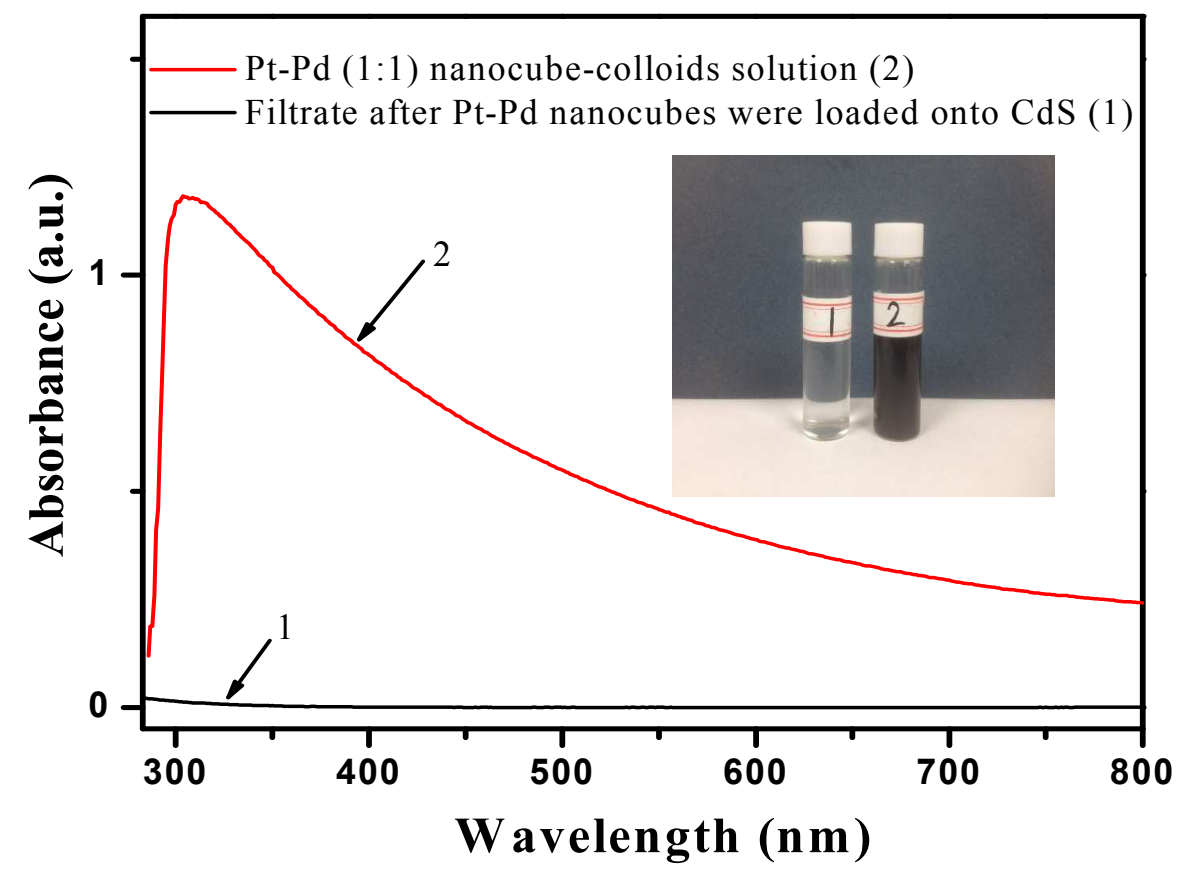

Fig. S1. $\mathrm{Pt}-\mathrm{Pd}(\mathrm{Pt}$ to $\mathrm{Pd}$ mass ratio = 1:1) nanocube-colloids solution before (2) and after (1) Pt-Pd nanocubes were loaded onto CdS. 


\section{Pt-Pd nanocube particles and size distributions}
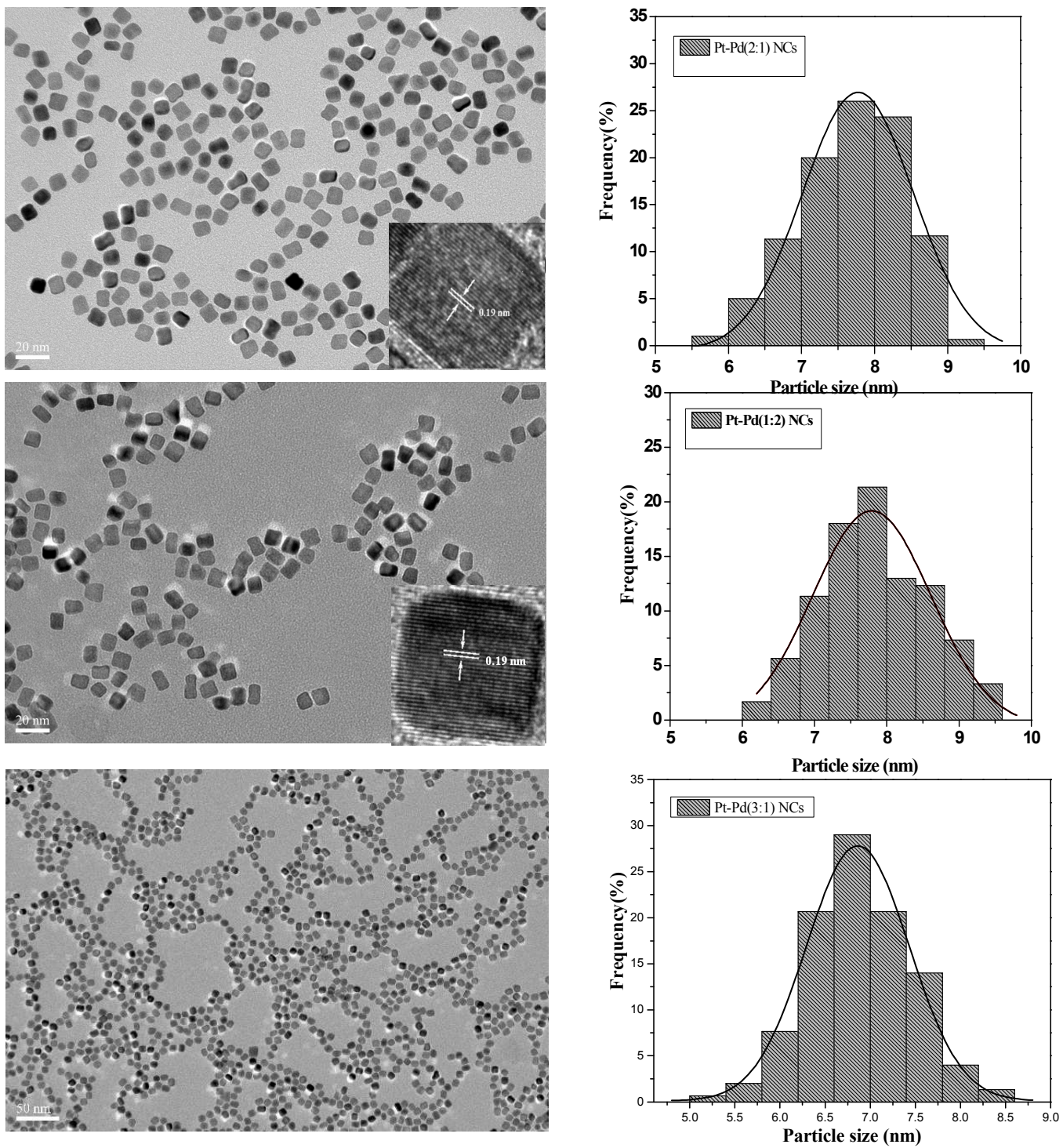

Fig. S2. TEM images and particle size distributions of Pt-Pd nanocubes. Top figures: $\mathrm{Pt}$ to $\mathrm{Pd}$ mass ratio $=2: 1$; Middle figures: $\mathrm{Pt}$ to $\mathrm{Pd}$ mass ratio $=1: 2$ ) and Bottom figures: $\mathrm{Pt}$ to $\mathrm{Pd}$ mass ratio $=3: 1$ alloys. The insets are the high resolution images of nanocubes. 
IV. TEM images of catalyst before and after hydrogen evolution

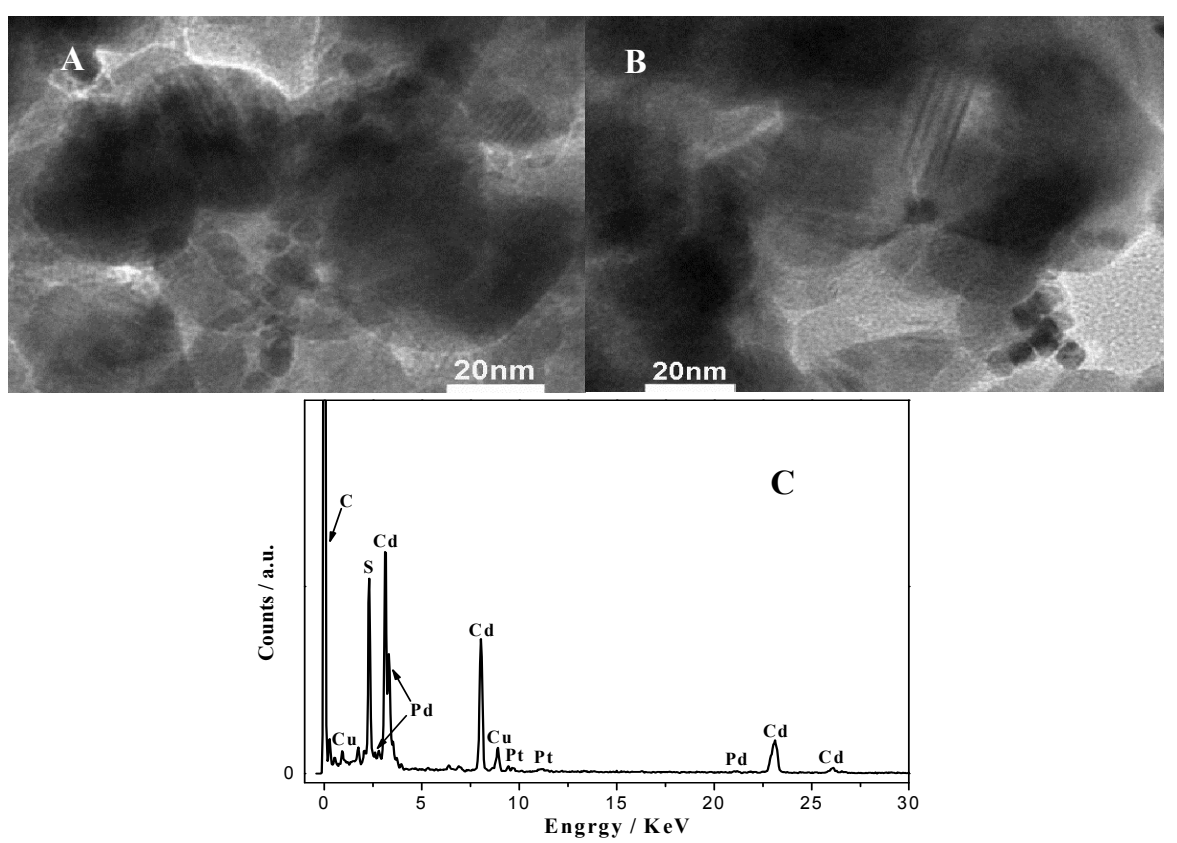

Fig. S3. TEM images of Pt-Pd (1:1) NOTs/CdS before (A) and after (B) photocatalytic hydrogen production. (C) Selected area energy dispersive spectroscopy (EDS) of a single metallic particle. 


\section{XAFS Measurements}
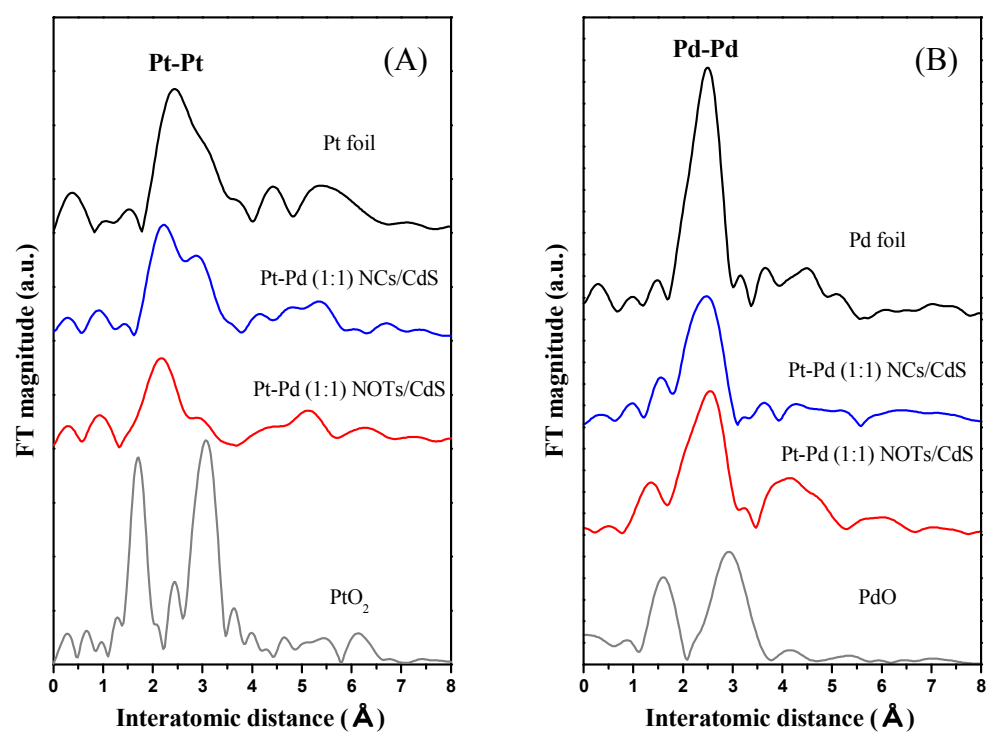

Fig. S4. (A) $\mathrm{Pt}_{2}$-edge FT-EXAFS spectra and (B) Pd K-edge FT-EXAFS spectra of Pt-Pd (1:1) NCs/CdS and Pt-Pd (1:1) NOTs/CdS after photocatalytc hydrogen production.

In Pt $\mathrm{L}_{3}$-edge FT-EXAFS spectra, both Pt-Pd (1:1) NCs/CdS and Pt-Pd (1:1) NOTs/CdS after photocatalytic hydrogen production showed a peak at around 2.1-2.4 $\AA$ that can be assigned to Pt-Pt bonding. In Pd K-edge FT-EXAFS spectra, both Pt-Pd $(1: 1) \mathrm{NCs} / \mathrm{CdS}$ and Pt-Pd (1:1) NOTs/CdS after photocatalytic hydrogen production showed a peak at around $2.5 \AA$, ascribed to Pd-Pd bonding. These results confirm that $\mathrm{Pt}$ and Pd are still in metallic state in Pt-Pd alloy nanocrystals after photocatalytic hydrogen production. 


\section{Turn-Over-Frequency (TOF) calculations}

Turn-Over-Frequency (TOF) calculation in this paper uses the method reported by Huang et al. (Nano lett. 2012, 12, 4265).

\section{(a). Mole of surface atoms in octahedral Pt-Pd nanocrystals}

Mole of surface atoms of octahedral Pt-Pd nanocrystal $=(\mathrm{Pt}$ atoms $+\mathrm{Pd}$ atoms on the surface of each octahedral Pt-Pd nanocrystal) / (Total moles of Pt atoms $+\mathrm{Pd}$ atoms in each Pt-Pd octahedral nanocrystal) $\times$ mole of octahedral Pt-Pd nanocrystals = $55.871 \mathrm{a}^{2} / 31.955 \mathrm{a}^{3} \times$ mole of octahedral Pt-Pd nanocrystals $=1.748 / \mathrm{a} \times$ mole of octahedral Pt-Pd nanocrystals

where, "a" is the edge length of a Pt-Pd octahedron particle

\section{(b) Mole of surface atoms in cubic Pt-Pd nanocrystals}

Mole of surface atoms of cubic Pt-Pd nanocrystal $=(\mathrm{Pt}$ atoms $+\mathrm{Pd}$ atoms on the surface of each cubic Pt-Pd nanocrystals) / (Total moles of Pt atoms $+\mathrm{Pd}$ atoms in each cubic Pt-Pd nanocrystal $) \times$ mole of cubic Pt-Pd nanocrystals $=80 \times b^{3} \div 67.797 x$ $\mathrm{b}^{3} \times$ mole of cubic Pt-Pd nanocrystals $=1.180 \div \mathrm{b} \times$ mole of cubic Pt-Pd nanocrystals where $b$ is the edge length of a Pt-Pd cube.

\section{(c) The photocatalytic turnover frequency (TOF)}

The photocatalytic turnover frequency (TOF) of Pt-Pd loaded CdS is defined as moles of hydrogen produced per surface mole of Pt-Pd metal atom per second. 